\title{
Classificação fuzzy da vulnerabilidade aos processos costeiros em Pontal do Paraná, Brasil
}

\author{
Nassau de Nogueira NARDEZ1,4, Rodrigo Mikosz GONÇALVES², Carlos Roberto SOARES³ \& Cláu- \\ dia Pereira KRUEGER ${ }^{4}$
}

(1) Faculdade de Engenharia Civil, Universidade Federal de Uberlândia. Av. João Naves de Ávila, 2121, CEP 38.400-902, Uberlândia, MG, Brasil. E-mail: nardeznassau@ufu.br.

(2) Programa de Pós-graduação em Ciências Geodésicas e Tecnologias da Geoinformação, Departamento de Engenharia Cartográfica, Universidade Federal de Pernambuco. Av. Acadêmico Hélio Ramos, s/n, CEP 50.740-530, Recife, PE, Brasil. E-mail: rodrigo.mikosz@ufpe.br.

(3) Centro de Estudos do Mar, Universidade Federal do Paraná. Av. Beira-Mar, s/n, Balneário Pontal do Sul, CEP 83.255-000, Pontal do Paraná, PR, Brasil. E-mail: crsoares@ufpr.br.

(4) Programa de Pós-graduação em Ciências Geodésicas, Departamento de Geomática, Universidade Federal do Paraná. Caixa Postal 19.001, Centro Politécnico, CEP 81.531-990, Curitiba, PR, Brasil. E-mail: ckrueger@ufpr.br.

Recebido em 06/2015. Aceito para publicação em 04/2016.

Versão online publicada em 31/05/2016 (www.pesquisasemgeociencias.ufrgs.br)

\begin{abstract}
Resumo - A classificação da vulnerabilidade aos processos costeiros considerando características tanto naturais quanto antrópicas serve de subsídio para a Gestão Integrada da Zona Costeira (GIZC). Nesta contribuição apresenta-se um sistema fuzzy para classificar a vulnerabilidade aos processos costeiros na região litorânea do Balneário de Pontal do Sul, PR, Brasil. A área de estudo foi dividida em três setores com características distintas, fornecendo diferentes valores para testar o sistema fuzzy. Este contém quatro variáveis de entrada: interferência antrópica, média de altura de ondas, amplitude de marés e a influência da desembocadura de um estuário. A classificação final do modelo apresenta como saída três variáveis linguísticas representando um determinado grau de vulnerabilidade, considerado como: estável, moderado e instável. Os resultados obtidos pelo sistema fuzzy, após a defuzzificação do sistema, são valores numéricos em uma escala de 0 a 8. Neste estudo, foram obtidos os seguintes valores: 5,9; 6,7; e 1,3 para os três setores avaliados, resultando numa classificação instável, instável e estável, respectivamente. Para corroborar a eficácia da modelagem, os resultados foram comparados com classificações existentes da área de estudo. Destaca-se que a metodologia é uma alternativa para contribuir com o papel do especialista e para tomada de decisão em estudos similares.
\end{abstract}

Palavras chave: Lógica fuzzy, linha de costa, vulnerabilidade aos processos costeiros, monitoramento costeiro, gestão integrada da zona costeira.

Abstract - Fuzzy classification of vulnerability to coastal processes in Pontal do Paraná, BRAZIL. The classification of the vulnerability for coastal processes considering both natural factors and anthropogenic interference can contribute for Integrated Coastal Zone Management (ICZM). This contribution develops a fuzzy system to classify the vulnerability of coastal processes in the coastal zone of Pontal do Sul, PR, Brazil. The study area was divided into three sectors with different characteristics, which provided diverse inputs to test this fuzzy system. Considered input variables are set as the follows: anthropogenic interference, average wave height, tidal range and the influence of the estuary in the region. The output of the final classification presents three linguistic labels, which represents three levels of vulnerability as stable, moderate and unstable. The results by the defuzzification in the fuzzy system are numerical values in a scale ranging from 0 to 8 . The following values have been obtained as 5.9, 6.7 and 1.3; for the three sectors indicating unstable, unstable and stable classification respectively. In order to corroborate the efficacy of modeling, the results were compared with existing classification of the study area. The method can be an alternative approach for the expert's role in decision-making in related studies.

Keywords: Fuzzy logic, shoreline, vulnerability of coastal processes, coastal monitoring, integrated coastal zone management. 


\section{Introdução}

As mudanças posicionais da linha de costa causadas por agentes naturais ou antrópicos caracterizam processos de progradação e erosão costeira, que podem ser consideradas como um dos principais problemas ambientais das Zonas Costeiras (ZC) (Souza, 2009; Gonçalves et al., 2012a, 2012b; Mendonça et al., 2014).

A erosão de largas faixas continentais se dá tanto por processos naturais quanto ações antrópicas (desmatamentos, construções, extração de areia ou remobilização de sedimentos, entre outros). Todo processo erosivo a curto, médio ou longo prazo causa transformações no ambiente do sistema praial, necessitando monitoramento e avaliações em relação à estabilidade da costa (Angulo, 1993a).

A estabilidade costeira está diretamente ligada à vulnerabilidade, suscetibilidade e ao risco à erosão ou progradação. De acordo com Angulo (1993a) a identificação e a classificação da costa em relação a sua estabilidade são importantes instrumentos de planejamento urbano ou territorial como, por exemplo, o estabelecimento de distâncias mínimas em relação à linha de costa para áreas construídas, e assim criar diretrizes e normas de ocupação da orla marítima (Muehe, 2005).

A erosão praial tem causado prejuízos em muitos países (Boruff et al., 2005; Rocha \& Diniz, 2011; Kent, 2013). De acordo com Gomes (2007) diversas metodologias e modelagens estão sendo desenvolvidas, relacionadas com vulnerabilidade e riscos costeiros à erosão. Kraus (1990) descreve a importância da modelagem numérica dos fenômenos que atuam nas regiões costeiras, discutindo suas aplicações no âmbito de planejamento integrado.

A vulnerabilidade costeira aos processos erosivos está relacionada a diversos fatores geológicos, geomorfológicos, oceanográficos, entre outros (Mazzer et al., 2008). As análises sobre este tema podem ter caráter local, regional, nacional e global. Os índices podem ser atribuídos a regiões de interesse, constituindo uma importante ferramenta para tomada de decisões (Mallmann \& Araújo, 2010).

Segundo Mazzer et al. (2008) o termo vulnerabilidade possui um amplo significado, não havendo um consenso em relação à sua conceituação, existindo divergências e convergências a este respeito. Harvey et al. (1999) afirmam que o conceito de vulnerabilidade inclui a susceptibilidade de uma área costeira às mudanças físicas produzidas pelas mudanças climáticas. Segundo Dutrieux et al. (2000) ela é uma associação de fatores de sensibilidade e riscos. A sensibilidade seria os fatores intrínsecos ao sistema natural, e os riscos estão relacionados aos efeitos antrópicos.

Estudos vêm sendo realizados para analisar ou determinar os níveis de vulnerabilidade e suscetibilidade em regiões costeiras como, por exemplo, Harvey et al. (1999), Boruff et al. (2005), Mazzer et al. (2008), Mallmann \& Araújo (2010), Raposeiro \& Ferreira (2010), Rudorff \& Bonetti (2010), Gonçalves et al. (2013), Silva et al. (2013), Mendonça et al. (2014), entre outros. Estes estudos apresentam uma gama de possibilidades para gerar indicativos que analisam a vulnerabilidade de regiões costeiras e, para tanto, fazem uso de diversos fatores, tais como os parâmetros: geomorfológicos (classificação da compartimentação do litoral), oceanográficos (clima de ondas), meteorológicos (regime de ventos e marés meteorológicas), astronômicos (marés lunisolares) e parâmetros antrópicos, marcados essencialmente por causas sociais e econômicas.

Os estudos de erosão, vulnerabilidade, susceptibilidade e a estabilidade em regiões costeiras podem se beneficiar da lógica fuzzy, que é fundamentada na teoria dos conjuntos difusos (Zadeh, 1965), sendo delineada como uma lógica precisa que trata da imprecisão e do raciocínio aproximado (Zadeh, 2008).

De acordo com Hanson et al. (2010), a lógica difusa permite um nível de abstração e generalização compatível com a escala regional de análise costeira. Diversos trabalhos têm utilizado a lógica fuzzy na determinação de vulnerabilidade como, por exemplo: erosão dos solos (Alves et al., 2005; Lobão et al., 2006), contaminação de águas subterrâneas (Dixon, 2005), predição de erosão de solo em bacias hidrográficas (Mitra et al., 1998) e influência de algumas variáveis consideradas ambientais (Cunha et al., 2011), contaminação de aquíferos freáticos (Lisbôa et al., 2010), erosão costeira (Rudorff \& Bonetti, 2010; Silva et al., 2013), entre outros.

Nesta contribuição, foi utilizado um sistema de inferência fuzzy como ferramenta para classificação de uma região costeira em relação à sua vulnerabilidade aos processos costeiros. A região de estudo situa-se no litoral do Estado do Paraná, Brasil, realizada no âmbito local (Balneário Pontal do Sul), a partir de fatores causadores de erosão ou progradação da linha de costa a partir de dados secundários, a saber: fatores físicos como ondas e marés, fatores antrópicos e fatores ligados à influência da desembocadura da região estuarina no oceano (Complexo Estuarino de Paranaguá - CEP). 
Os resultados foram confrontados com a classificação existente da área (Angulo, 1993a) a fim de corroborar ou não os resultados obtidos pela lógica fuzzy.

\section{2 Área e métodos}

\subsection{Localização da área}

A área do presente estudo localiza-se no Balneário Pontal do Sul, PR, ela foi dividida em três setores distintos (Fig. 1) segundo suas características em relação ao Complexo Estuarino de Paranaguá (CEP). O Setor (I) é o mais interno, dominado pela ação das correntes da desembocadura, o setor (II) é intermediário e o setor (III) é o mais externo, exposto à ação direta das ondas oceânicas.
A região de estudo apresenta regime de micro-marés semi-diurno, com correntes de deriva litorânea que predominam de Sul para Norte (Angulo, 1993b). Esse sentido de deriva reflete o predomínio da energia das ondas provenientes de Sul-Sudeste, relacionadas com a passagem de frentes-frias (Angulo, 1996).

A desembocadura do CEP é caracterizada como um delta de maré vazante, onde atuam um canal central principal de vazante e canais marginais de enchente (Angulo, 1992). Estas feições mostram grandes variações de sua morfologia em curtos espaços de tempo, causando importantes mudanças na área costeira adjacente, como intensos processos de erosão e deposição nas praias, bem como modificações na posição da linha de costa (Lamour et al., 2003).

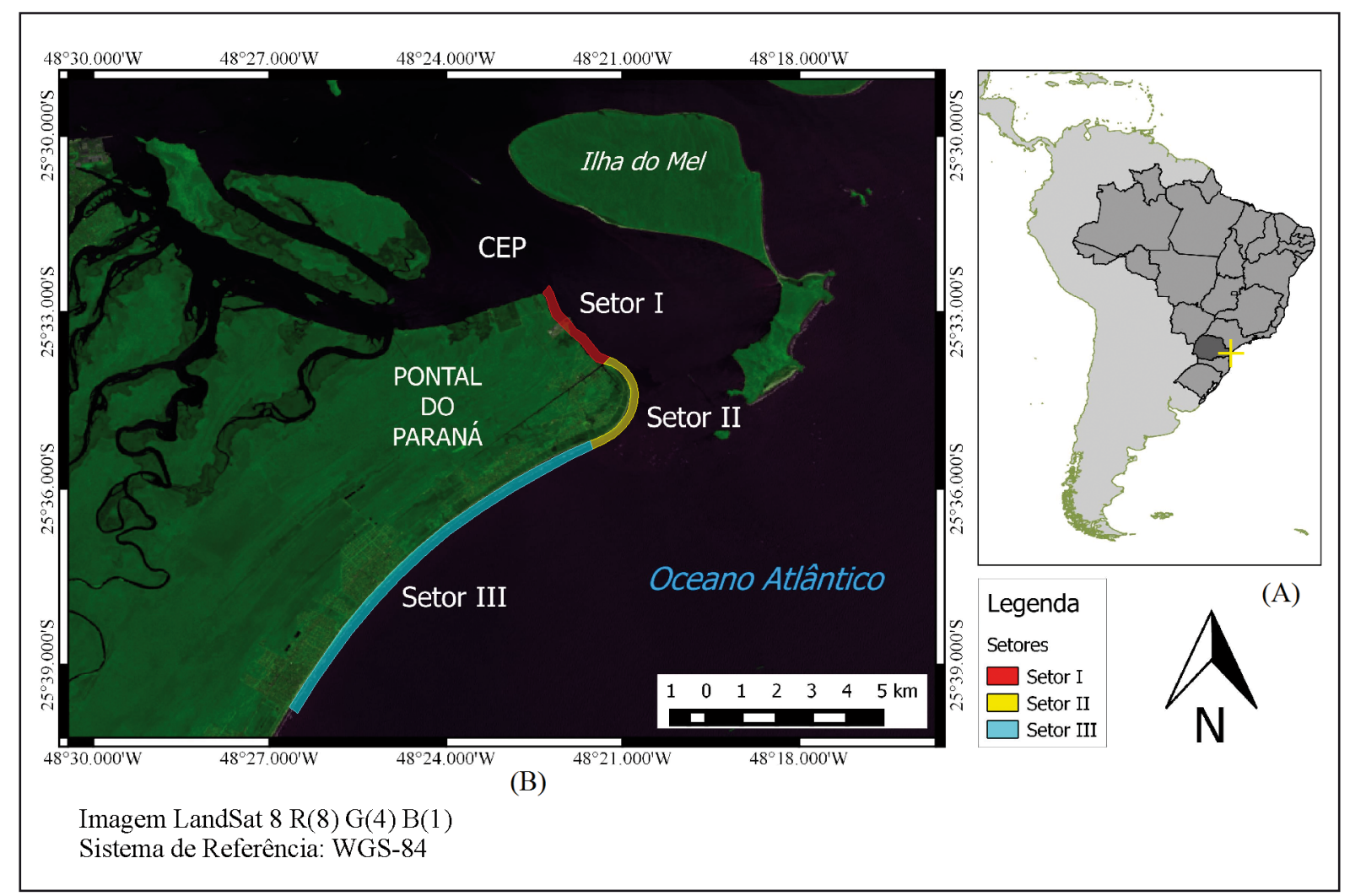

Figura 1. Mapa de localização. A) Destaque para o estado do Paraná, indicando a localização aproximada da área de estudo (modificada a partir de USGS, 2015); B) Área de estudo, que abrange os setores I, II e III, no município de Pontal do Paraná, Região Sul do Brasil.

\subsection{Conjuntos fuzzy}

A lógica fuzzy é baseada na teoria dos conjuntos fuzzy, ocupa-se do uso de valores nebulosos para captar o significado das palavras, do raciocínio humano e tomadas de decisões. É um método para codificar e aplicar o conhecimento de uma forma que reflete com precisão o entendimento de um especialista em problemas difíceis e complexos (Negnevitsky, 2005).
Desenvolvida por Lotfi A. Zadeh (Zadeh, 1965) esta teoria define um conjunto fuzzy simplesmente como um conjunto de limites difusos (Negnevitsky, 2005), ou seja, os limites dos conjuntos se sobrepõem.

Seja $X$ um conjunto universo e seus elementos denotados por $x$. Na teoria fuzzy, um subconjunto $A$ de $X$ é definido pela função $\mu A(x)$ chamada função de pertinência do subconjunto $A$, que assume valores em um intervalo $[0,1]$ : 


$$
\mu_{A}(x): X \rightarrow[0,1]
$$

sendo que $\mu_{A}(x)^{\prime}=1$ se $x$ está totalmente em $A, \mu_{A}(x)=0$ se $x$ não está em $A$ e $0<\mu_{A}(x)<1$ se $x$ está parcialmente em $A$.

O subconjunto fuzzy $A$ em $X$ é então representado por um conjunto de pares ordenados:

$A=\left\{\mu_{A}(x) / x\right\}$

Para qualquer elemento $x$ do universo $X$, a função de pertinência $\mu A(x)$ fornece o grau com que $x$ pertence ao conjunto $A$, sendo este grau um valor entre 0 e 1 , chamado grau de pertinência de $x \operatorname{em} A$.

Então $A$ é considerado um subconjunto fuzzy de $X$ se, e somente se:

$$
A=\left\{x, \mu_{A}(x)\right\} ; x \in X, \mu_{A}(x): X \rightarrow[0,1]
$$

Verifica-se, no entanto que o subconjunto $A$ é definido em termos da função de pertinência.

\subsubsection{Sistemas de inferência baseado em regras fu- zzy $(S B R F)$}

A lógica fuzzy fornece subsídios para construção dos sistemas de inferência nebulosos (Mendel, 1995). Um dos métodos mais comum e aplicado neste trabalho é o denominado Mamdani, um método para a captura de um determinado conhecimento especializado. Permite descrever a experiência de forma intuitiva, semelhante à humana, tendo a capacidade de capturar o conhecimento considerado especialista por meio de regras fuzzy (Negnevitsky, 2005). Esta classe de sistema é amplamente utilizada em problemas de modelagem, controle e classificação (Jafelice et al., 2005).

0 processo de inferência fuzzy é constituído por quatro etapas: a fuzzificação das variáveis de entrada, a criação da base de regras, o processamento dos dados das variáveis pela máquina de inferência e a defuzzificação da variável de saída. A figura 2 ilustra as etapas do processo de um sistema de inferência fuzzy.

De acordo com Ross (2010) a fuzzificação é o processo que torna os valores das entradas em valores nebulosos (fuzzy). Se a forma da incerteza da variável surge por causa da sua imprecisão, ambiguidade ou por sua vagueza, então, a variável é provavelmente difusa e pode ser representada por uma função de pertinência. A fuzzificação é o processo que define os conjuntos fuzzy de uma variável por meio de suas funções de pertinência em relação ao universo desta variável. Para cada um destes conjuntos fuzzy são atribuídos variáveis linguísticas para representá-los.

A fuzzificação das entradas crisp (lógica tradicional) em conjuntos fuzzy é necessária para ativar as regras que são em termos de variáveis linguísticas, as que têm conjuntos fuzzy associados a elas (Mendel, 1995).

As funções de pertinência definem o grau de pertinência de uma entrada em um ou mais conjuntos fuzzy. Os conjuntos fuzzy são grupos qualitativos definidos por suas respectivas funções de pertinência e são identificados por suas variáveis linguísticas. De acordo com Gomide et al. (1995), a principal função das variáveis linguísticas é fornecer uma maneira sistemática para uma caracterização aproximada de fenômenos complexos ou mal definidos. Esse tipo de descrição linguística empregada por seres humanos permite o tratamento de sistemas com alto grau de complexidade para serem analisados por mecanismos matemáticos convencionais.

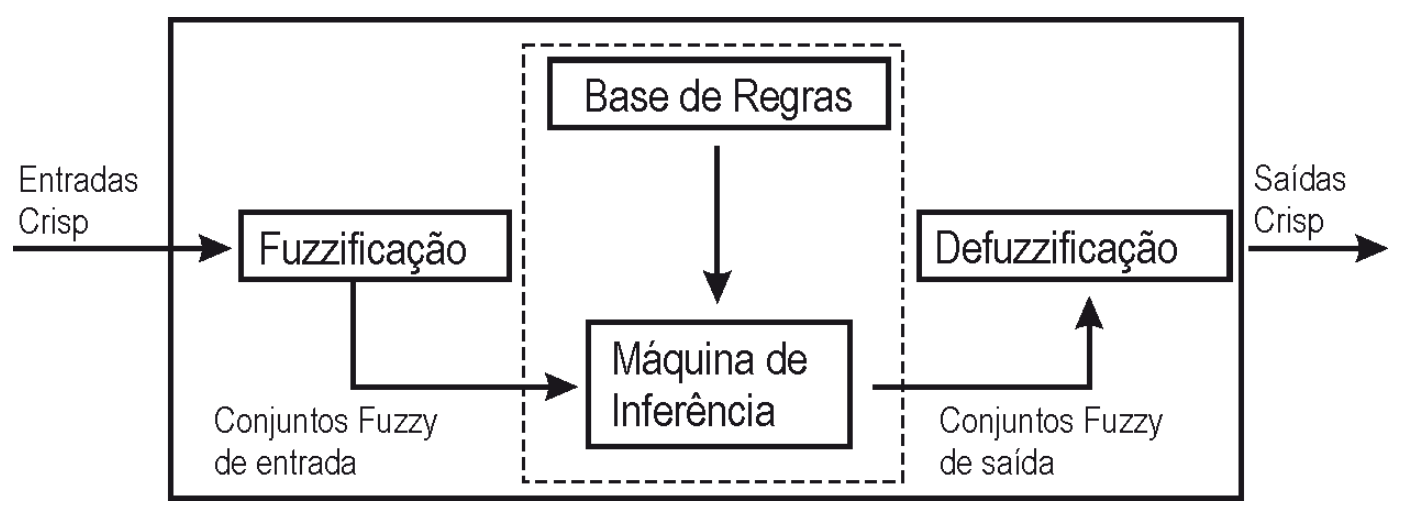

Figura 2. Esquema do sistema de inferência baseado em regras fuzzy (Adaptado de Mendel, 1995). 


\subsubsection{Base de regras}

A base de regras constitui a base de conhecimentos, de acordo com Shaw \& Simões (1999), sendo que as regras são criadas empiricamente, a partir de valores ou de sentenças linguísticas fornecidas por um especialista. A base de regras é composta por uma coleção de proposições fuzzy na forma "se-então", que descreve a dependência de uma variável em relação à outra. Em cada base de regras adota-se o operador matemático mínimo para o conectivo lógico "e" e o operador máximo para o conectivo "ou". As sentenças "se-então" são modeladas pela aplicação do mínimo e agregadas através do máximo (Amendola et al., 2005). Estas regras permitem implementar um algoritmo computacional por meio da articulação de estratégias para ação das regras descritas por termos linguísticos imprecisos.

\subsubsection{Máquina de inferência fuzzy}

De acordo com Jafelice et al. (2005), nesta componente utiliza-se técnicas de raciocínio apro- ximado para traduzir matematicamente cada proposição fuzzy, e os operadores matemáticos são selecionados para definir a relação fuzzy que modela a base de regras.

Ainda em Jafelice et al. (2005), verifica-se que para o método de Mamdani, uma regra "se" (antecedente) "então" (consequente) é definida pelo produto cartesiano fuzzy dos conjuntos fuzzy que compõem o antecedente e o consequente da regra. Este método agrega as regras por meio do operador lógico " $o u$ ", que é modelado pelo operador máximo e, em cada regra, o operador lógico "e" é modelado pelo operador mínimo, como no exemplo:

Regra 1: Se ( $x$ é A1 e yé B1) então ( $z$ é $C 1$ );

Regra 2: Se ( $x$ é $A 2$ e yé B2) então ( $z$ é $C 2$ ).

A figura 3 ilustra a geração de uma saída real $z$ de um sistema de inferência tipo Mamdani a partir das entradas $x$ e $y$ reais e a regra de composição max-min. A saída $z$ é obtida pela defuzzificação (seção 2.2.4) do conjunto fuzzy de saída $C=C_{1}^{\prime} U C_{2}^{\prime}$ (Jafelice et al., 2005).

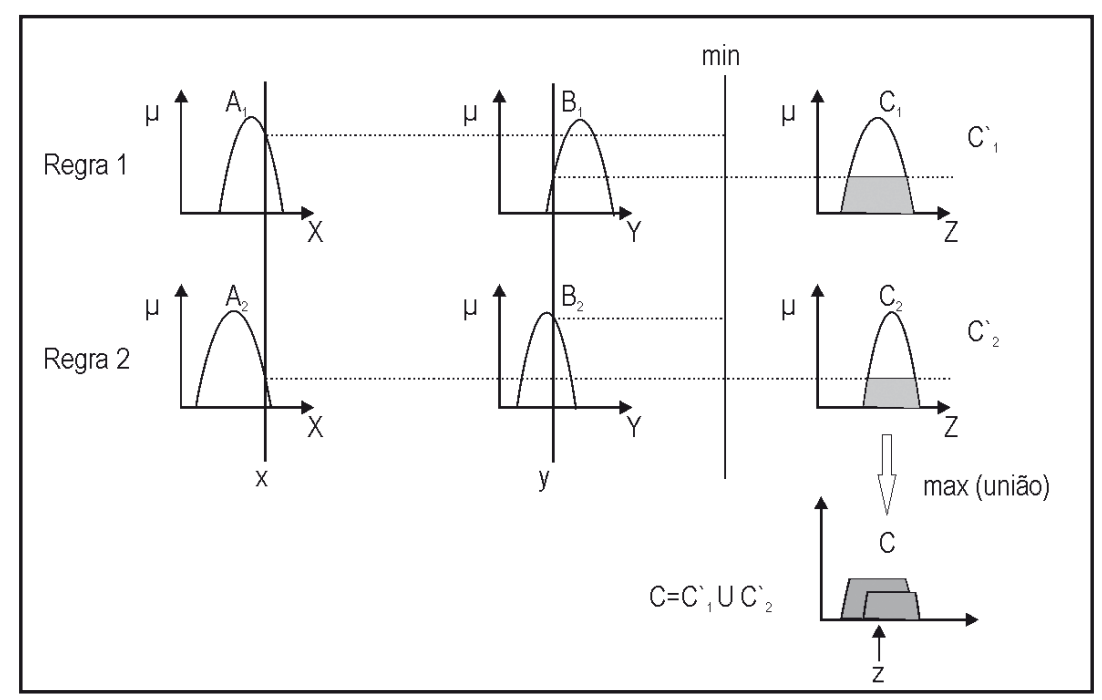

Figura 3. Composição max-min pelo método de Mamdani (Fonte: Jafelice et al., 2005).

\subsubsection{Defuzzificação}

O último passo no processo de inferência fuzzy é defuzzificação. A imprecisão ajuda a avaliar as regras, mas o resultado final de um sistema $f u$ zzy é um número crisp (convencional). 0 processo de defuzzificação transforma o conjunto fuzzy de saída agregado em um único número (Negnevitsky, 2005).

Para obter um número crisp de saída que a represente, deve-se escolher um método de defuzzificação. 0 método escolhido neste trabalho foi o centro de gravidade, pois é semelhante à média ponderada para distribuição de dados, com a di- ferença que os pesos são os valores $C\left(z_{i}\right)$ que indicam o grau de compatibilidade do valor $z_{i}$ com o conceito modelado pelo conjunto fuzzy $C$ (Jafelice et al., 2005).

Matematicamente o centro de gravidade $(G)$ pode ser expresso como:

$G(C)=\frac{\sum_{i=0}^{n} u_{i} C\left(z_{i}\right)}{\sum_{i=0}^{n} C\left(z_{i}\right)}$

Este método calcula o ponto onde uma linha vertical iria cortar o conjunto agregado em duas massas iguais (Negnevitsky, 2005). 


\subsection{Metodologia aplicada}

\subsubsection{Definição das variáveis}

Os fatores erosivos considerados na região de estudo e que foram utilizados como variáveis de entrada no processo de inferência fuzzy são:

(i) Interferência antrópica: segundo Muehe (2006) nos casos onde se verificam processos erosivos, efeitos antrópicos não podem ser descartados, tais como os provocados por dragagens e despejo de materiais dragados. No litoral paranaense o fator antrópico mais significativo é a ocupação inadequada da área costeira. Na região em estudo destaca-se o canal artificial do Departamento Nacional de Obras de Saneamento (DNOS), aberto entre as décadas de 1950 e 1960, e as dragagens periódicas no canal de acesso ao Porto de Paranaguá (canal da Galheta), localizado na porção intermediária do CEP.

(ii) Ondas: de acordo com Muehe (2001) a amplitude de resposta aos processos de erosão, transporte e acumulo da mobilização sedimentar depende do clima de ondas e do grau de exposição do segmento costeiro considerado. Bigarella et al. (1978) afirmam que, com a passagem de sistemas frontais, principalmente no inverno, ocorre um aumento da energia das ondas, retirando os sedimentos da costa e alterando a configuração da praia de forma significativa;

(iii) Marés: da mesma forma que a ação das ondas, as correntes de maré retiram e depositam os sedimentos na costa. Elas são geradas a partir da subida e descida das marés em regiões costeiras, transportam sedimentos ao longo da costa, podendo ser depositados nas praias (Bird, 1996). A classificação das variações de maré proposta por Davies (1964) foi utilizada no presente trabalho, sendo subdividida em áreas de micromarés (quando a maré é menor que $2 \mathrm{~m}$ ), mesomarés (entre $2 \mathrm{e}$ $4 \mathrm{~m}$ ) e macromarés (maior que $4 \mathrm{~m}$ ); e

(iv) Influência da desembocadura do CEP: a influência da desembocadura do CEP diz respeito às correntes de maré enchente e vazante do estuário. Verifica-se que a maior influência encontrada localiza-se no setor II pelo fato de causar uma barreira hidráulica na maré vazante em conjunto com a corrente litorânea, que tem sentido sul-norte. Neste setor de hidrodinâmica complexa, existem inúmeros bancos arenosos que compõem o delta de maré vazante do CEP, e que por sua vez interagem permanentemente com as marés, correntes de marés, ondas e correntes litorâneas.

\subsubsection{Fuzzificação}

Na tabela 1 são apresentadas as características gerais do processo de fuzzificação das variáveis de entrada do sistema. A fuzzificação das variáveis de entrada foi realizada utilizando as funções de pertinência trapezoidais (Tab. 1), que permitem a definição de um intervalo do universo de discurso da variável com pertinência total ao conjunto representado, o que, neste caso, possibilita uma melhor representação das variáveis consideradas.

As variáveis de entrada foram definidas por suas variáveis linguísticas e representadas por seus valores linguísticos, o tipo das funções de pertinência, o domínio das variáveis, e os valores que definem as funções em relação aos valores de pertinência.

A figura 4 representa a fuzzificação da variável de entrada interferência antrópica, onde os valores do domínio estão representados no eixo das abscissas, e no eixo das ordenadas são representados o grau de pertinência $\left(\mu_{(I A)}\right)$ da variável em cada conjunto (Baixa, Média e Alta) variando de 0 a 1, onde 0 representa a não pertinência e 1 representa a pertinência total ao conjunto.

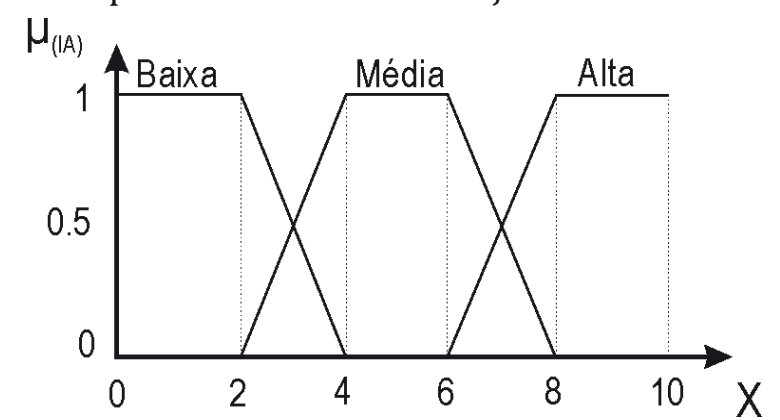

Figura 4. Variável interferência antrópica (IA) e seus conjuntos fuzzy.

Para a variável interferência antrópica determinou-se um domínio de 0 a 10 de forma adimensional, possibilitando caracterizar o grau de interferência antrópica em cada um dos setores. 0 conjunto fuzzy denominado Baixa varia de 0 a 4 , onde a função possui pertinência total $\left(\mu_{(I A)}=1\right.$ em Baixa) entre os valores de 0 a 2 , a partir deste ponto a função decresce linearmente até pertinência nula em 4. A função do conjunto fuzzy Média inicia-se com pertinência nula em 2 e a partir deste ponto aumenta linearmente até atingir pertinência total em 4, no intervalo de 4 a 6 possui pertinência total $\left(\mu_{(A I)}=1\right.$ em Média), então decresce linearmente até a pertinência nula em 8. Por fim, a função do conjunto fuzzy Alta inicia-se em $6 \mathrm{com}$ pertinência nula atingindo pertinência total em 8 até 10. 
Tabela 1. Características gerais da fuzzificação dos dados de entrada do sistema.

\begin{tabular}{|c|c|c|c|c|}
\hline \multicolumn{5}{|c|}{ Variável Linguística: Interferência antrópica (adimensional) } \\
\hline \multicolumn{2}{|c|}{ Domínio: 0 a 10} & \multicolumn{3}{|c|}{ Intervalo numérico } \\
\hline Valor linguístico & Tipo de função de pertinência & $\mu=0$ & $\mu=1$ & $\mu=0$ \\
\hline Baixa & Trapezoidal & - & $0-2$ & 4 \\
\hline Média & Trapezoidal & 2 & $4-6$ & 8 \\
\hline Alta & Trapezoidal & 6 & $8-10$ & - \\
\hline \multicolumn{5}{|c|}{ Variável Linguística: Média de altura de ondas (m) } \\
\hline \multicolumn{2}{|c|}{ Domínio: 0 a 8} & \multicolumn{3}{|c|}{ Intervalo numérico } \\
\hline Valor linguístico & Tipo de função de pertinência & $\mu=0$ & $\mu=1$ & $\mu=0$ \\
\hline Baixa & Trapezoidal & - & $0-1$ & 3 \\
\hline Média & Trapezoidal & 1 & $3-4$ & 6 \\
\hline Alta & Trapezoidal & 4 & $6-8$ & - \\
\hline \multicolumn{5}{|c|}{ Variável Linguística: Amplitude de marés (m) } \\
\hline \multicolumn{2}{|c|}{ Domínio: 0 a 6} & \multicolumn{3}{|c|}{ Intervalo numérico } \\
\hline Valor linguístico & Tipo de função de pertinência & $\mu=0$ & $\mu=1$ & $\mu=0$ \\
\hline Baixa & Trapezoidal & - & $0-1$ & 2 \\
\hline Média & Trapezoidal & 1 & $2-4$ & 5,5 \\
\hline Alta & Trapezoidal & 4 & $6-8$ & - \\
\hline \multicolumn{5}{|c|}{ Variável Linguística: Influência da desembocadura do CEP (adimensional) } \\
\hline \multicolumn{2}{|c|}{ Domínio: 0 a 10} & \multicolumn{3}{|c|}{ Intervalo numérico } \\
\hline Valor linguístico & Tipo de função de pertinência & $\mu=0$ & $\mu=1$ & $\mu=0$ \\
\hline Baixa & Trapezoidal & - & $0-2$ & 4 \\
\hline Média & Trapezoidal & 2 & $4-6$ & 8 \\
\hline Alta & Trapezoidal & 6 & $8-10$ & - \\
\hline
\end{tabular}

As principais interferências antrópicas consideradas na área de estudo foram: construção de um píer, construção de um canal de navegação e ainda a dragagem no CEP do canal de acesso ao porto de Paranaguá.

A figura 5 apresenta a fuzzificação da variável de entrada Ondas (médias dos valores obtidos por Nemes, 2011). Observa-se que para altura de ondas de 0 a $1 \mathrm{~m}$ a pertinência no conjunto fuzzy Bai$x a$ é igual a 1, a partir deste ponto decresce linearmente até atingir pertinência 0 para ondas de $3 \mathrm{~m}$. O conjunto fuzzy Média inicia-se com ondas de $1 \mathrm{~m}$ aumentando linearmente até atingir pertinência 1 no conjunto fuzzy Média para ondas de $3 \mathrm{~m}$ até ondas de $4 \mathrm{~m}$, e então decresce linearmente até a pertinência 0 para ondas de $6 \mathrm{~m}$ (Fig. 5). Finalmente, o conjunto fuzzy Alta inicia-se para ondas de $4 \mathrm{~m}$ aumentando linearmente a partir deste ponto até atingir pertinência 1 neste conjunto para ondas de $6 \mathrm{~m}$ ou maiores.A figura 6 apresenta a fuzzificação da variável de entrada Maré (baseada na classifica- ção de Davies, 1964). Observa-se que para marés de 0 a 1,8 m a pertinência no conjunto fuzzy Baixa é igual a 1. A partir deste ponto decresce linearmente até atingir pertinência 0 para marés de 2,2 m. 0 conjunto fuzzy Média inicia-se com marés de 1,8 $\mathrm{m}$ aumentando linearmente até atingir pertinência 1 no conjunto fuzzy Média para marés de 2,2 $\mathrm{m}$ até $3,8 \mathrm{~m}$ e então decresce linearmente até a pertinência 0 para marés de $4,2 \mathrm{~m}$. Finalmente, o conjunto fuzzy Alta inicia-se para marés de 3,2 m aumentando linearmente a partir deste ponto até atingir pertinência 1 neste conjunto para marés de $4,2 \mathrm{~m}$ ou maiores.

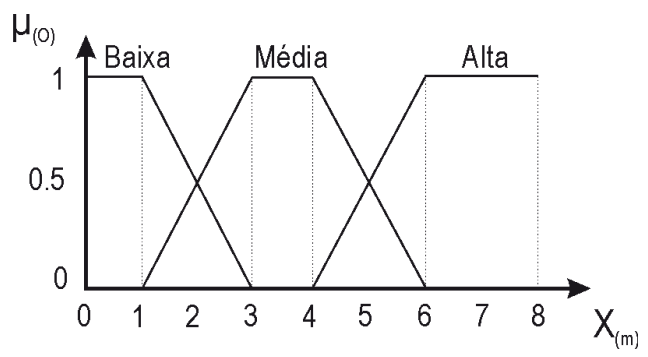

Figura 5. Variável ondas (0) e seus conjuntos fuzzy. 
Verifica-se na figura 7 que para a variável influência da desembocadura do estuário determinou-se um domínio de 0 a 10 de forma adimensional permitindo caracterizar a influência das correntes de maré para cada um dos setores. 0 conjunto fuzzy Baixa varia de 0 a 4, onde a função possui pertinência total $\left(\mu_{(I D E)}=1\right.$ em Baixa $)$ entre os valores de 0 a 2; a partir deste ponto a função decresce linearmente até pertinência nula em 4 . A função do conjunto fuzzy Média inicia-se com pertinência nula em 2 e aumenta linearmente até atingir pertinência total em 4, no intervalo de 4 a 6 onde possui pertinência total $\left(\mu_{(I D E)}=1\right.$ em Média), então decresce linearmente até a pertinência nula em 8. A função do conjunto fuzzy Alta inicia-se em 6 com pertinência nula atingindo pertinência total em 8 até 10.

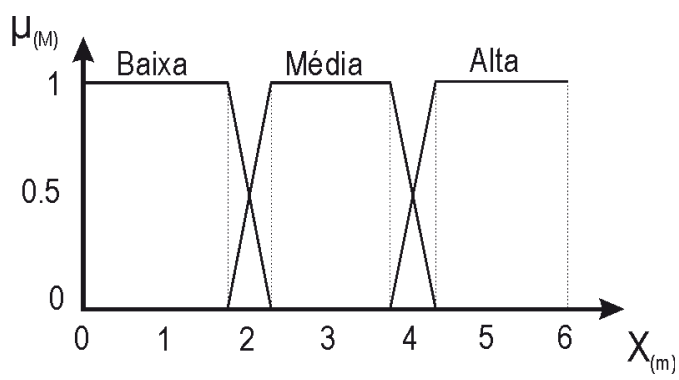

Figura 6. Variável marés (M) e seus conjuntos fuzzy.

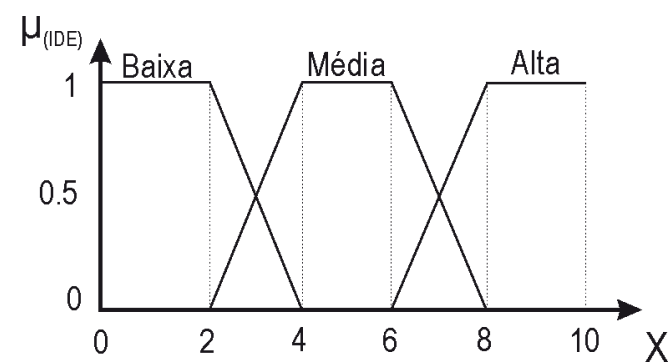

Figura 7. Variável influência da desembocadura do Estuário (IDE) e seus conjuntos fuzzy.

\subsubsection{Saída do sistema baseado em regras fuzzy}

Na tabela 2 são apresentadas as características do conjunto de saída. Verifica-se que foi estabelecido um Domínio de 0 a 8 , utilizou-se de funções trapezoidais para definição dos conjuntos, e os valores linguísticos adotados na classificação costeira foram: Estável, Moderado e Instável conforme ilustra a figura 8.

\subsubsection{Valores de entrada do sistema baseado em re-} gras fuzzy

Os valores de entrada inseridos no sistema de inferência fuzzy pelo método de Mamdani foram obtidos a partir de dados secundários da área de estudo, tendo sido utilizados como referência: Angulo (1993a, 1993b, 1996), Lamour et al. (2003), Calliari et al. (2003), Ignácio et al. (2004), Veiga et al. (2005), Muehe (2006) e Nemes (2011).

A partir destes trabalhos foi possível obter os valores médios de ondas (dados obtidos em Nemes, 2011) e marés (dados obtidos em Veiga, 2005), e serviram de subsídios para determinar os valores da interferência antrópica e da influência da desembocadura. Foram também efetuadas visitas in situ, para verificação dos valores atribuídos à interferência antrópica para cada um dos setores.

Na tabela 3 apresentam-se os valores das variáveis linguísticas de entrada do sistema de inferência para cada um dos setores analisados.

No Setor I, o valor atribuído para a interferência antrópica foi considerado alto, pelo fato de haver dragagens periódicas, bem como a existência de um canal artificial e a construção de um píer, que alteraram a hidrodinâmica local. Para o valor da influência da desembocadura considerou-se o fato de existirem bancos arenosos nesta região, pertencentes ao delta de maré vazante do CEP, que lhe confere maior complexidade na interação entre as ondas provindas de offshore (plataforma continental), correntes de marés de enchente e vazante, e as correntes de deriva litorânea.

Para o Setor II considerou-se a dragagem do canal de acesso ao porto de Paranaguá como o principal fator de influência antrópica, bem como uma maior influência da desembocadura, pelo fato de haver deposição de sedimentos naquela região causada pelas correntes de maré vazante do estuário.

Para o Setor III, os valores da interferência antrópica e da influência da desembocadura foram mínimos, por não haver na região fatores que caracterizem essas duas variáveis.

Desta forma, estes valores, que representam as características de cada setor da região, para cada uma das variáveis, foram inseridos na entrada do sistema de inferência, baseado em regras fuzzy, de forma que a saída fornece a classificação em relação à estabilidade costeira de cada um dos setores analisados.

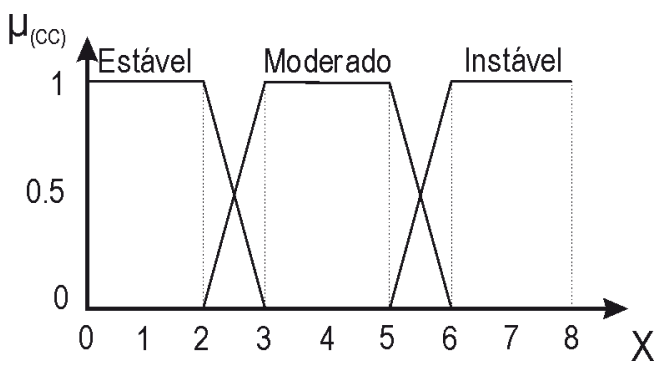

Figura 8. Variável classificação costeira (CC) e seus conjuntos fuzzy. 
Tabela 2. Características gerais da fuzzificação da saída do sistema fuzzy.

\begin{tabular}{c|c|c|c|c}
\hline \multicolumn{2}{c|}{ Estabilidade Costeira Domínio: 0 a 8} & \multicolumn{3}{c}{ Valores fuzzy limites } \\
\hline Variáveis linguísticas & Tipo da Função de pertinência & $\mu=0$ & $\mu=1$ & $\mu=0$ \\
\hline Estável & Trapezoidal & - & $0-2$ & 3 \\
Moderado & Trapezoidal & 2 & $3-5$ & 6 \\
Instável & Trapezoidal & 5 & $6-8$ & - \\
\hline
\end{tabular}

Tabela 3. Valores de entrada do sistema fuzzy.

\begin{tabular}{c|c|c|c}
\hline Classes & Setor I & Setor II & Setor III \\
\hline Interferência antrópica & 8 & 5 & 1 \\
Ondas (m) & 0,25 & 0,5 & 1,48 \\
Marés (m) & 1,4 & 1,4 & 1,4 \\
Influência da Desembocadura do CEP & 3,5 & 7,5 & 1 \\
\hline
\end{tabular}

\section{Resultados e discussão}

\subsection{Setor I}

Para este setor, obteve-se um valor de 5,86 no processo de inferência fuzzy. Isto denota uma classificação em relação à instabilidade da costa, uma transição de moderado para instável. 0 resultado indica que este setor possui um grau de pertinência de 0,28 no conjunto moderado e um grau de pertinência de 0,72 no conjunto instável, lembrando que a pertinência abrange um conjunto que varia de 0 a 1.

Na classificação realizada por Angulo (1993a, 1993b) foram utilizadas fotografias aéreas dos anos de 1952, 1955 e 1980, na escala de 1:25.000, e de 1963 na escala 1:70.000, uma imagem TM LANDSAT5 de 1985 e cartas náuticas. Com base na variação da linha de costa este setor obteve a classificação considerada como moderado. 0 principal fator que levou a esta classificação foi a interferência antrópica, ou seja, a construção do canal artificial do DNOS que interrompeu parte da deriva longitudinal de sedimentos, provocando erosão a noroeste do canal e sedimentação a sudoeste (Angulo, 1993b). Porém, mais recentemente, foi construído um píer (Fig. 9), tendo como consequência tendência de erosão acentuada a sudoeste desta obra.

\subsection{Setor II}

0 valor obtido pela inferência fuzzy para o Setor II foi de 6,7 podendo ser classificado como instável, o que é corroborado pela classificação realizada por Angulo (1993a).

Este setor foi classificado como instável possi- velmente por ser uma região intermediária (entre a desembocadura do estuário e o mar aberto). A linha de costa pode apresentar variações de dezenas até centenas de metros. Um dos motivos pode estar relacionado com o fato de que os sedimentos transportados pelas correntes de deriva litorânea encontram uma barreira hidráulica causada pelas correntes de maré vazante do estuário, provocando a deposição de sedimentos, e consequentemente, gerando progradação da linha de costa, conforme ilustra as posições temporais da linha de costa vista em Ignácio et al. (2004). Na figura 10 é possível observar os bancos de areia adjacentes a esta região, pertencentes ao delta do CEP.

\subsection{Setor III}

De acordo com Angulo (1993a) o Setor (III) possui características externas ao estuário, expostas à ação das ondas, sendo classificado como estável, apresentando variações da linha de costa inferiores a $10 \mathrm{~m}$, no período de 1954 a 1980, com predomínio da sedimentação nas últimas décadas. Este setor é identificado como uma praia oceânica ou de mar aberto.

Como a dinâmica da região é dominada pelas ondas e pelas correntes de deriva litorânea, este setor aparentemente encontra-se em equilíbrio dinâmico, não apresentando variações significativas na configuração da linha de costa (Angulo, 1993a).

Esta classificação corrobora o resultado obtido pela inferência fuzzy sendo o valor obtido igual a 1,30. Este valor indica um grau de pertinência igual a 1 no conjunto estável, sendo a classificação para o Setor III estável uma vez que o setor está totalmente contido neste conjunto. 


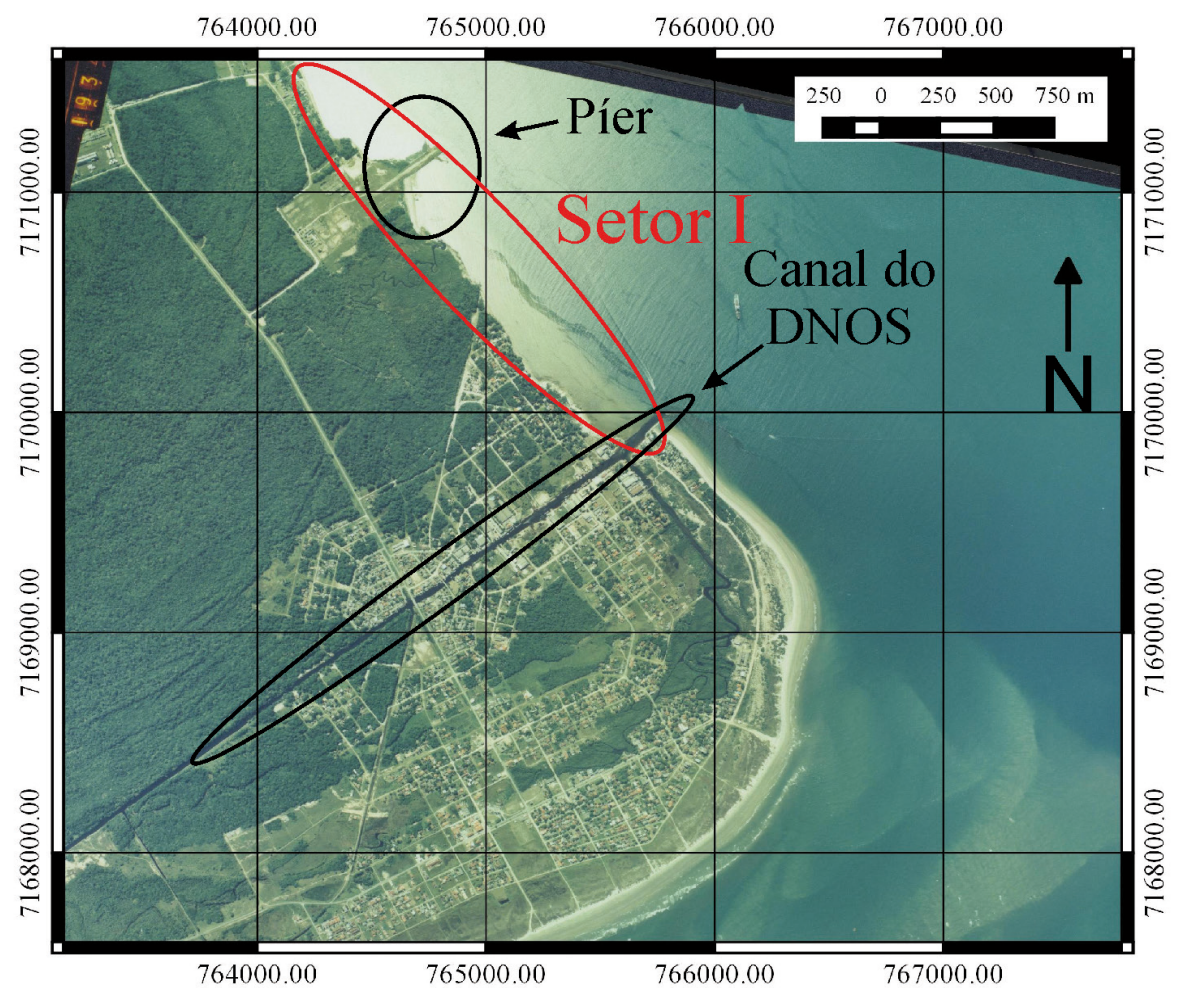

Foto aérea. Escala: 1/25000

Sistema de Referência: SIRGAS2000

Figura 9. Construção do Píer localizado no Setor I (Modificado de USGS, 2015).

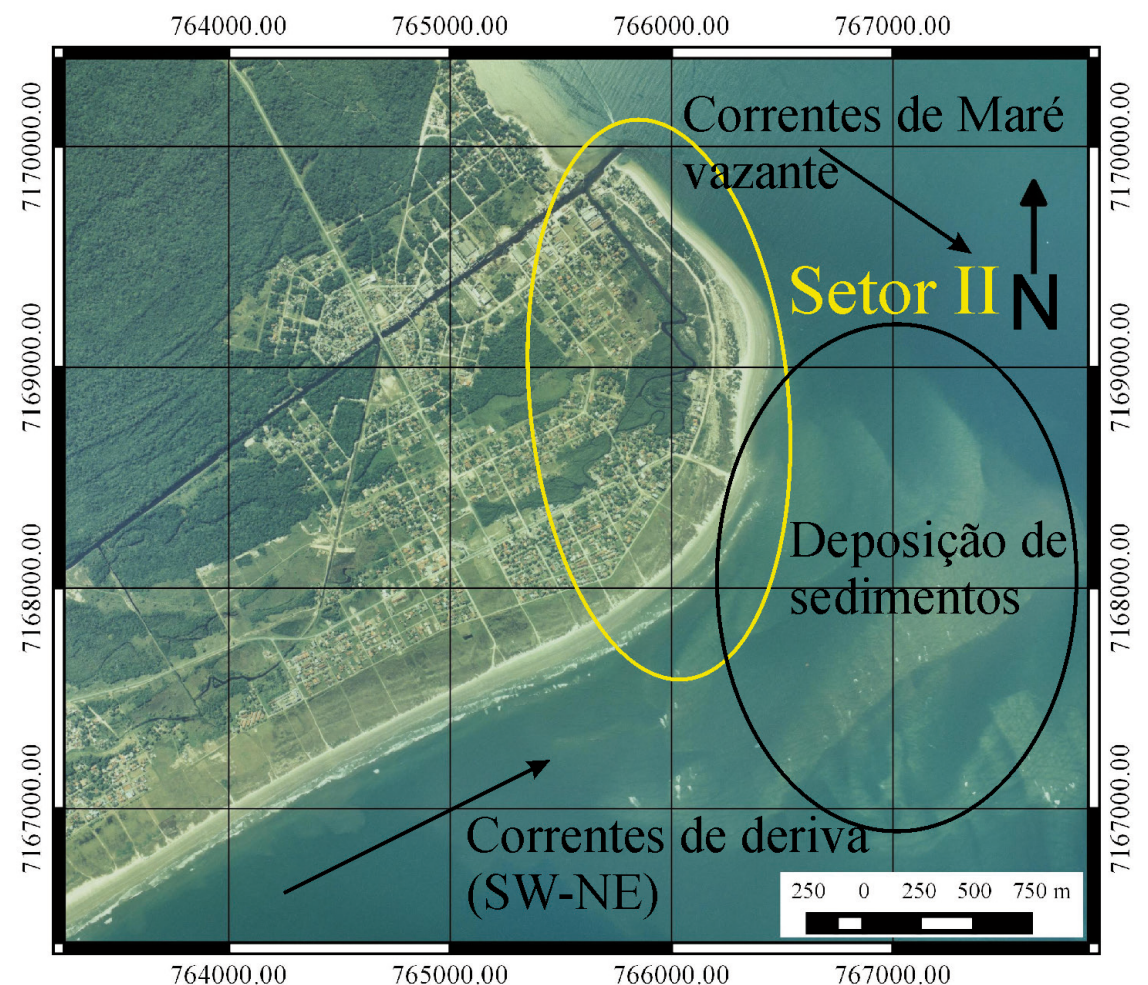

Foto aérea. Escala: 1/25000

Sistema de Referência: SIRGAS2000

Figura 10. Bancos de areia localizados no Setor II (Modificado de USGS, 2015). 


\section{Conclusões}

O sistema desenvolvido de classificação em relação aos processos costeiros utilizando conceitos da lógica fuzzy teve empregadas quatro variáveis de entrada: interferência antrópica na região, média de altura de ondas, amplitude de marés e a influência da desembocadura de um estuário na região estudada.

Depois de configurado, o sistema fuzzy foi testado com valores para as variáveis obtidos na literatura para três setores com características diferentes. Como resultado a modelagem apresenta um valor numérico baseado na teoria dos conjuntos fuzzy que ao mesmo tempo é convertido em uma variável linguística, representando a classificação do setor em estudo. Sendo assim, obtiveram-se os respectivos valores e classificações: 5,9 - instável (setor I); 6,7 - instável (setor II) e 1,3 - estável (setor III).

Para validar a classificação obtida pelo sistema de inferência fuzzy foram comparados os produtos obtidos com uma classificação independente apresentada por pesquisas realizadas na área. 0 resultado desta comparação apresentou para o setor I a classificação moderada pelo especialista e instável pela modelagem proposta. Destaca-se que a teoria fuzzy pode representar duas classes ao mesmo tempo, contendo um valor de pertinência para cada, neste caso foi obtido um valor de pertinência de 0,28 para o conjunto moderado e 0,72 para o conjunto instável, o que indica a transição da classificação do setor I de moderado para instável. Para o setor II a classificação obtida foi instável pelo modelo e pelas pesquisas realizadas na área. E para o setor III também houve coincidência em ambas as classificações, sendo estas, estáveis.

Verifica-se que a classificação do Setor I obtida pela inferência fuzzy foi influenciada pela alta interferência antrópica no local, uma vez que, além das constantes dragagens no canal de acesso ao estuário houve a construção de um píer, aumentando o processo erosivo da costa naquela zona do litoral. Salienta-se a capacidade do sistema fuzzy em considerar novos fatores, como este mencionado, uma vez que não havia tal construção na época da classificação existente.

Para os setores I e II, a tendência atual é marcada pelas oscilações com forte retrogradação e progradação, respectivamente, nas praias influenciadas pela desembocadura do estuário (Muehe, 2006), bem como a continuação da tendência erosiva no setor I originada pelo Canal do DNOS e pela construção do píer, caracterizando a instabilidade costeira. Ainda há as ações antrópicas próximas aos setores, fatores absorvidos pelo sistema fuzzy e que corroboram os resultados obtidos tendendo à instabilidade costeira.

Em relação ao setor III, a tendência é a estabilidade costeira, por ser uma região de praia oceânica com ocupação recuada em relação à linha de costa (Muehe, 2006), de forma que o principal fator erosivo atuante são as ações diretas das ondas. Observa-se que o sistema fuzzy corrobora esta tendência.

Para finalizar destacam-se algumas considerações: (i) novos parâmetros de entrada podem ser configurados na inferência fuzzy para classificar quanto à vulnerabilidade aos processos costeiros, por exemplo, regime de ventos e marés meteorológicas, entre outros; (ii) uma classificação quanto à vulnerabilidade aos processos costeiros deve ser refeita no decorrer do tempo, devido às mudanças climáticas e antrópicas, salientando a importância do monitoramento costeiro; (iii) neste experimento verificou-se que os parâmetros de entrada mais influentes para a área de estudo foram a interferência antrópica e a influência da desembocadura do estuário.

Agradecimentos - Ao CNPq pela bolsa de doutorado do primeiro autor, ao Centro de Estudos do Mar (CEM) da UFPR, ao Laboratório de Cartografia Costeira (LACCOST) da UFPE e ao Laboratório de Geodésia Espacial e Hidrografia (LAGEH) da UFPR.

\section{Referências}

Alves, C.A., Florenzano, T.G. \& Pereira, M.N. 2005. Mapeamento de áreas urbanizadas com imagens Landsat e classificação baseada em objeto. Revista Brasileira de Cartografia, 62: 189-198.

Amendola, M., Souza, A.L. \& Barros, L.C. 2005. Manual do uso da teoria dos conjuntos Fuzzy no MATLAB 6.5. FEAGRI \& IMECC/UNICAMP, 44p.

Angulo, R.J. 1992. Geologia da planície costeira do Estado do Paraná. São Paulo, 334p. Tese de Doutorado, Programa de Pós-graduação em Geologia Sedimentar, Instituto de Geociências, Universidade de São Paulo.

Angulo, R.J. 1993a. A ocupação urbana do litoral paranaense e as variações da linha de costa. Boletim Paranaense de Geociências, 41: 73-81.

Angulo, R.J. 1993b. Classificação da costa paranaense com base na sua dinâmica, como subsídio a ocupação da orla litorânea. In: SIMPÓSIO SUL-BRASILEIRO DE GEOLOGIA,5, Curitiba. Boletim de resumos $e$ programa: 69-70.

Angulo, R.J. 1996. Natural dynamics and land-use on the coast of Paraná, Brazil: background and prospective. Anais da Academia Brasileira de Ciências, 68(3): 383-388.

Bigarella, J.J., Beker, R.D., de Matos, D.J. \& Werner, A.A. 
1978. Serra do mar e a porção oriental do estado do Paraná. Um problema de segurança ambiental e nacional (contribuição à geografia, geologia e ecologia regional). Governo do Estado do Paraná, Secretaria de Estado do Planejamento - Associação de Defesa e Educação Ambiental (ADEA). Curitiba. 248p.

Bird, E.C.F. 1996. Beach management. New York, John Wiley and Sons Ltd, 281p.

Boruff, B.J., Emrich, C. \& Cutter, S.L. 2005. Erosion hazard vulnerability of US coastal counties. Journal of Coastal Research, 21(5): 932-942.

Calliari, L.J., Muehe, D., Hoefel, F.G. \& Toldo Jr., E.J. 2003. Morfodinâmica praial: uma breve revisão (Beach morphodynamics: a brief review). Revista Brasileira de Oceanografia, 51: 63-78.

Cunha, R.C. da, Dupas, F.A., Pons, N.A.D. \& Tundisi, J.G. 2011. Análise da influência das variáveis ambientais utilizando inferência fuzzy e zoneamento das vulnerabilidades. Estudo do caso da bacia hidrográfica do Ribeirão do Feijão, São Carlos - SP. Geociências, 30(3): 399-414.

Davies, J.L. 1964. A morphogenic approach to world shorelines. Zeitschrift für Geomorphologie, 8: 127142.

Dixon, B. 2005. Groundwater vulnerability mapping: a GIS and fuzzy rule based integrated tool. Applied Geography, 25(4): 327-347.

Dutrieux, E., Canovas, S., Denis, J., Hénocque, Y., Quod, J.P. \& Bigot, L. 2000. Guidelines for vulnerability mapping of coastal zones in the Indian Ocean. n. 38. Paris, INFREMER/UNESCO, International Oceanographic Commission Manuals and Guide, 41p.

Gomes, F.V. 2007. A gestão da zona costeira portuguesa. Revista da Gestão Costeira Integrada. 7(2): 83-95.

Gomide, F.A.C., Gudwin, R.R. \& Tanscheit, R. 1995. Conceitos fundamentais da teoria de conjuntos fuzzy, lógica fuzzy e aplicações. In: INTERNATIONAL FUZZY SYSTEMS ASSOCIATION WORLD CONGRESS, 6, Proceedings..., TUTORIALS - IFSA95, p. 01-38.

Gonçalves, R.M., Awange, J., Krueger, C.P., Heck, B. \& Coelho, L.A. 2012a. Comparison between three short-term shoreline prediction models. Ocean \& Coastal Management, 69: 102-110.

Gonçalves, R.M., Awange, J. \& Krueger, C.P. 2012b. GNSS-based monitoring and mapping of shoreline position in support of planning and management of Matinhos/PR (Brazil). Journal of Global Positioning Systems, 11: 156-168.

Gonçalves, R.M., Pacheco, A.D.P., Tanajura, E.L.X. \& Silva, L.M. 2013. Urbanização costeira e sombreamento na praia de Boa Viagem, Recife-PE, Brasil. Revista de Geografia Norte Grande, 54: 241-255.

Hanson, S., Nicholls, R.J., Balson, P., Brown, I., French, J.R., Spencer, T. \& Sutherland, W. J. 2010. Capturing coastal geomorphological change within regional integrated assessment: an outcome-driven Fuzzy logic approach. Journal of Coastal Research, 26(5): 831-842.

Harvey, N., Clouston, B. \& Carvalho, P. 1999. Improving coastal vulnerability assessment methodologies for Integrated Coastal Zone Management: an approach from South Australia. Australian Geographical Studies, 37: 50-69.

Ignácio, G.M., Rebuli, K.B., Krug, L.A., Kotler, L., Tel, M.P. \& Patchineelam, S.M. 2004. Consequências da intervenção antrópica na Zona Costeira: um exemplo do litoral do Paraná. Brasil. Disponível em: <http://www.uesb.br/anpuhba/artigos/anpuh_II/ gabriela_m_ignacio.pdf>. Acesso em: 20 de jan. 2015.

Jafelice, R.S.M., Barros, L.C. de \& Bassanezi, R.C. 2005. Teoria dos conjuntos Fuzzy com aplicações. São Paulo, ed. Plêiade, 66p.

Kent, S.T. 2013. Restoration of the Barataria basin Barrier Islands. Journal of Undergraduate Engineering Research and Scholarship, 1: 1-8.

Kraus, N.C. (Ed.). 1990. Shoreline change and storm-induced beach erosion modeling: a collection of seven papers. Mississippi, Final Report, Miscellaneous Paper CERC-90-2 (disponível em http://bhl-china. org/bhldatas/pdfs/s/shorelinechanges00krau. pdf\#page=9 > . Acesso em: 13 abr. 2016.

Lamour, M.R., Noernberg, M.A., Quadros, C.J., Odreski, L.L. \& Soares, C.R. 2003. Erosão na desembocadura da Baía de Paranaguá e sua relação com o assoreamento do Canal da Galheta. In: CONGRESSO DE ASSOCIAÇÃO BRASILEIRA DE ESTUDOS DO QUATERNÁRIO, 9, Recife, 4p.

Lisbôa, E.G., Mendes, R.L.R. \& Barp, A.R.B. 2010. Mapeamento da vulnerabilidade de aquíferos freáticos baseado em Lógica Fuzzy. In: CONGRESSO BRASILEIRO DE ÁGUAS SUBTERRÂNEAS, 16, São Luís, 18p.

Lobão, J.S.B., Rocha, W.de J.S.daF. \& Silva, A.B. 2006. Utilização de Lógica Fuzzy na modelagem da vulnerabilidade à erosão no município de Morro do Chapéu-BA. In: SIMPÓSIO REGIONAL DE GEOPROCESSAMENTO E SENSORIAMENTO REMOTO, 3, Anais...Aracaju. 6p.

Mallmann, D.L.B. \& Araújo, T.C.M. 2010. Vulnerabilidade do Litoral Sul de Pernambuco à erosão. Tropical Oceanography, 38(2): 129-151.

Mazzer, A.M., Dillenburg, S.R. \& Souza, C.R.G. 2008. Proposta de método para análise de vulnerabilidade à erosão costeira no sudeste da ilha de Santa Catarina, Brasil. Revista Brasileira de Geociências, 38(2): 278294.

Mendel, J.M. 1995. Fuzzy logic systems for engineering: a tutorial. Proceedings of the IEEE, 83(3): 345-377.

Mendonça, F.J.B., Gonçalves, R.M., Awange, J., Silva, L.M. \& Gregório, M.N. 2014. Temporal shoreline series analysis using GNSS. Boletim de Ciências Geodésicas, 20(3): 701-719.

Mitra, B., Scott, H.D., Dixon, J.C. \& Mckimmey, J.M. 1998. Applications of fuzzy logic to the prediction of soil erosion in a large watershed. Geoderma, 86(3): 183209.

Muehe, D. 2001. Critérios Morfodinâmicos para o Estabelecimento de Limites da Orla Costeira para fins de Gerenciamento. Revista Brasileira de Geomorfologia, 2(1): 35-44.

Muehe, D. 2005. Aspectos gerais da erosão costeira no Brasil. Mercator - Revista de Geografia da UFC, 4(7): 97-110.

Muehe, D. (org.). 2006. Erosão e progradação do litoral 
brasileiro. Ministério do Meio Ambiente, Brasília, DF, Brasil, 476p.

Negnevitsky, M. 2005. Artificial intelligence. A guide to intelligent systems. 2 ed. England, Addison Wesley, $440 \mathrm{p}$.

Nemes, D.D. 2011. Caracterização das ondas de superfície em duas profundidades da plataforma interna do estado do Paraná. Pontal do Sul, 130p. Dissertação de Mestrado, Programa de Pós-graduação em Sistemas Costeiros e Oceânicos, Centro de Estudos do Mar, Universidade Federal do Paraná.

Raposeiro, P.D. \& Ferreira, J.C.R. 2010. A análise da vulnerabilidade e do risco de inundação como ferramenta de apoio à gestão dos territórios litorais sob pressão urbana. Pluris: Planejamento urbano regional integrado e sustentável. Conference Paper, 12p. Disponível em <https://www.researchgate.net/publication/268447126>. Acesso em: 13 abr. 2016.

Rocha, G.C. \& Diniz, M.T.M. 2011. Implicações da erosão costeira em atividades econômicas na praia da Caponga - Cascavel - Ceará. Scientia Plena, 7(2): 1-9.

Ross, T.J. 2010. Fuzzy logic with engineering applications. 3 ed. London, John Wiley and Sons Ltd, 606p.

Rudorff, F.M. \& Bonetti, J. 2010. Avaliação da suscetibilidade à erosão costeira de praias da ilha de Santa Catarina. Brazilian Journal of Aquatic
Science and Technology, 14(1): 9-20.

Shaw, I.S. \& Simões, M.G. 1999. Controle e modelagem Fuzzy. São Paulo, Ed. Edgard Blucher Ltda, 200p.

Silva, L.M., Gonçalves, R.M., Lira, M.M.S. \& Pereira, P.S. 2013. Modelagem fuzzy aplicada na detecção da vulnerabilidade à erosão costeira. Boletim de Ciências Geodésicas, 19: 746-764.

Souza, C.R. de G. 2009. Coastal erosion and the coastal zone management challenges in Brazil. Journal of Integrated Coastal Zone Management, 9(1): 17-37.

USGS. U.S. Geological Survey. 2015. Maps, Imagery, and Publications. Washington, NASA-USGS. Disponível em: <http://www.usgs.gov/pubprod/>. Acesso em: $15 / 03 / 2015$.

Veiga, F.A., Angulo, R.J., Marone, E., Brandini, F.P. \& Soares, C.R. 2005. Padrões de transporte de sedimentos baseado em três programas geradores de vetores de tendências de transporte a partir de parâmetros granulométricos na porção sul do delta de desembocadura do Complexo Estuarino de Paranaguá - Sul do Brasil. Boletim Paranaense de Geociências, 57: 75-87.

Zadeh, L.A. 1965. Fuzzy Sets. Information and Control, 8(3): 338-353.

Zadeh, L.A. 2008. Is there a need for fuzzy logic? Information Sciences, 178: 2751-2779.

Man. 566.

Editores: Ana Maria P. Mizusaki \& Maria do Carmo

Lima e Cunha. 
